Fleischmann, M., S. Pons, and G. Preparata, Possible theories of cold fusion. Nuovo Cimento Soc. Ital. Fis. A, 1994. 107: p. 143.

\title{
Possible Theories of Cold Fusion
}

\author{
M. Fleischmann $\left({ }^{1}\right)$, S. Pons $\left({ }^{2}\right)$ and G. Preparata $\left({ }^{3}\right)$ \\ ${ }^{1}$ ) Department of Chemistry, University of Southampton - Highfield Southampton S09 5NH, UK \\ $\left({ }^{2}\right)$ Department of Chemistry, University of Utah - Salt Lake City, UT 84.109 \\ $\left({ }^{3}\right)$ Dipartimento di Fisica dell'Università - Via Celoria 16, 20122 Milano \\ (ricevuto l' 11 Giugno 1993; approvato il 4 Agosto 1993)
}

\begin{abstract}
Summary. - We review some of the key facts in the phenomenology of Pdhydrides usually referred to as «cold fusion». We conclude that all theoretical attempts that concentrate only on few-body interactions, both electromagnetic and nuclear, are probably insufficient to explain such phenomena. On the other hand we find good indications that theories describing collective, coherent interactions among elementary constituents leading to macroscopic quantum-mechanical effects belong to the class of possible theories of those phenomena.
\end{abstract}

PACS 12.20.Ds - Specific calculations and limits of quantum electrodynamics.

PACS 52.55.Pi - Confinement in fusion experiments (including $\alpha$-particle effects, scaling laws, etc.).

\section{Introduction.}

In the preliminary paper on the subject which has become known as «cold fusion», we pointed out that it was the strange behaviour of hydrogen and its isotopes dissolved in metal lattices [1-6] which led us to pose the question whether it might be possible to induce nuclear reactions of deuterium by electrochemical compression into host lattices such as palladium [7-9]. The evidence which has been accumulated during the past two years has confirmed the initial observations that this is indeed possible and that the processes differ remarkably from the fusion paths of high-energy deuterons in the gas phase, e.g. see the reviews [10-13].

In sect. 1 we outline a few of the key facts about the dissolution of hydrogen and its isotopes in palladium as well as some simple deductions which can be drawn from these observations, the scenario of the topic. In sect. 2 we illustrate that, whereas it is possible to explain individual peculiarities of the behaviour by making ad hoc assumptions about the properties of individual hydrogen (deuterium) atoms or ions in the lattice, these ad hoc assumptions are in conflict with each other. Such descriptions must therefore be classified under the heading of «impossible theories». It is our view that a comprehensive description of these conventional properties must be based on the collective behaviour of the ensemble of hydrogen (deuterium) ions which form a dense plasma in the host lattice $[14,15]$. This collective behaviour must be invoked even without considering the strange nuclear processes induced by the lattice but seems to set the scene for the consideration of these processes.

In sect. 2 of this paper we outline in turn some of the key facts about the nuclear processes observed to date which all seem to highlight the remarkable differences between «cold» and «hot fusion». 
The interpretation of these facts is considered in sect. 3 and in sect. 4, which extends the modelling of the conventional equilibrium properties to that of nuclear processes in lattices. Here we show that reasonable formulations of both the ingoing and outgoing channels must again be based on the collective behaviour of dense plasmas. In sect. 5 we point out that all phenomena which depend on the coherent behaviour of systems, of which «cold fusion» is but one example, will require the proper consideration of the relevant macroscopic wave functions.

\section{1. - The strange behaviour of hydrogen and its isotopes dissolved in metal host lattices.}

As has been noted in the introduction, we illustrate the strange behaviour of hydrogen and its isotopes dissolved in metal host lattices by the example of palladium. The most immediately obvious peculiarity is the very high concentration of the dissolved species which lies in the range 60-100 molar for cathodically polarized electrodes. Here, the lower limit will apply to electrodes at low cathodic overpotentials where the system has been fully charged in the initial condition of the $\beta$-phase. As we have noted previously [7], it would be expected that the concentration of dissolved hydrogen would increase at high cathodic overpotentials possibly approaching or even exceeding an $\mathrm{H}: \mathrm{Pd}$ ratio of 1 ; this expectation has now received fragmentary confirmation and, at this limit, the concentration of dissolved hydrogen will be $\sim 100$ molar.

The hydrogen in the palladium lattice is present as protons $[16,17]$ and the second surprising feature is that the extremely dense proton plasma is present in an electron concentration of 600$1000 \mathrm{M}$ [4]. It is immediately evident that any simple two-body model of the system would demand the instantaneous formation of highly compressed hydrogen and, since this does not take place, we are driven to the conclusion that a satisfactory explanation of the stable existence of the dense plasma must be based on an appropriate many-body model.

Further important features of the system are the very high diffusion coefficients (of the order $10^{-7} \mathrm{~cm}^{2} \mathrm{~s}^{-1}$ for hydrogen in the a Pd-H phase) and the fact that the diffusion coefficients are in the order $D_{\mathrm{D}^{+}}>D_{\mathrm{H}^{+}}>D_{\mathrm{T}^{+}}$rather than in the order $D_{\mathrm{H}^{+}}>D_{\mathrm{D}^{+}}>D_{\mathrm{T}^{+}}$which would be expected on the basis of any simple classical model of diffusion [4]. We also have to take note of the fact that the systems $\beta$-PdH and $\beta$-PdD show an inverse isotope effect in the transition temperatures to the superconducting state and, indeed, that these superconductors must be classified as being «lukewarm» [18].

An observation which had an important influence on the decision to initiate work on «cold fusion» was the measurement of very high equilibrium H/D separation factors for hydrogen and deuterium dissolution into palladium cathodes at high negative overpotentials [3]. The interpretation of these high separation factors requires the assumption that the species behave as classical oscillators in the lattice, i.e. that they are virtually unbound. Although this conclusion is really in the nature of a deduction setting the scenario for the system, we believe that this deduction is so immediate that the description of the dissolved protons and deuterons as classical oscillators can really be classified as a basic property of the system. We note here also that the separation factors for the discharge reaction are also so high that they require the assumption of an «unbound» transition state [3]. We comment further on the interpretation of this observation in sect. 5 .

The high mobility of the isotopic species in the lattice [16,17], the high equilibrium separation factors into the lattice and in the discharge reaction and a number of other features which require 
very loose binding in the lattice are clearly in conflict with simple deductions of the properties of the system which can be based for example on the thermodynamics of the dissolution processes. Thus the simplest form of Born-Haber cycle based on the assumption of the formation of atomic hydrogen in the lattice

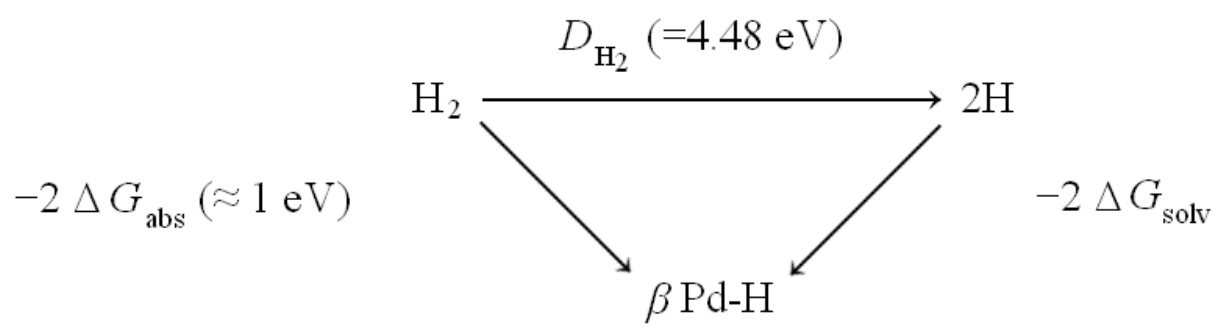

requires a «solvation energy» of the atoms of at least $2.74 \mathrm{eV}$. The chemical potential of the dissolved species can be raised by compression of the gas and we have noted elsewhere that the application of high cathodic overpotentials can lead to even larger changes in the chemical potential, at least of the order $0.8 \mathrm{eV}$. Such changes require an increase of the solvation energy to say $\sim 3.54 \mathrm{eV}$ and/or a marked change in the absorption energy so much so that, based on an extrapolation of the known heats of absorption of $\mathrm{H}$ in $\mathrm{Pd}$ [19] to high $\mathrm{H} / \mathrm{Pd}$ loading ratios, the absorption may become endothermic. This scenario has had an important bearing on the protocol which we specified for the experiments and has an equally important bearing on the outcome of experiments in which samples loaded with deuterium are subjected to changes of temperature.

However, we do not believe that the scenario in which the dissolved species are present as atoms rather than ions can be correct since it is inconsistent with many of the observations and, especially, the stable existence of the system at very high species concentration. The modified Born-Haber cycle based on the dissolution of $\mathrm{H}^{+}$gives a solvating energy in the lattice of at least $11.7 \mathrm{eV}$ which is raised further by the application of high cathodic overpotentials (which must,

$$
\begin{aligned}
& D_{\mathrm{H}_{2}}(=4.48 \mathrm{eV})
\end{aligned}
$$

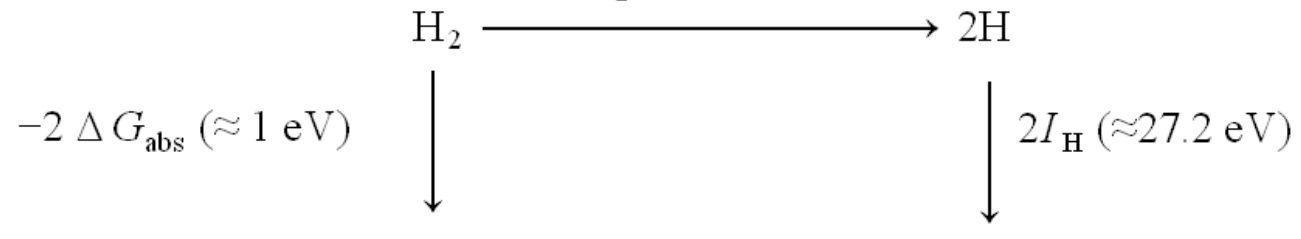

$$
\begin{aligned}
& \beta \mathrm{Pd}-\mathrm{H}^{+}-\mathrm{e} \longleftarrow 2 \mathrm{H}^{+}+2 \mathrm{e} \\
& -2 \Delta G_{\mathrm{solv}}+2 \varphi(=9.28 \mathrm{eV})
\end{aligned}
$$

furthermore, lead to a reduction in the work function and, therefore, to a yet further increase in $\Delta G_{\text {solv }}$ ) (see fig. 1). In terms of the chemistry of the system we have to ask how can a proton affinity in the range $11.7-13 \mathrm{eV}$ arise. This magnitude is so large that we are driven to the conclusion that the dense plasma is in some form of collective state. We address the resolution of the problem of the strong binding of the protons (deep energy wells) coupled to their behaviour as loosely bound species in sect. 3 . 


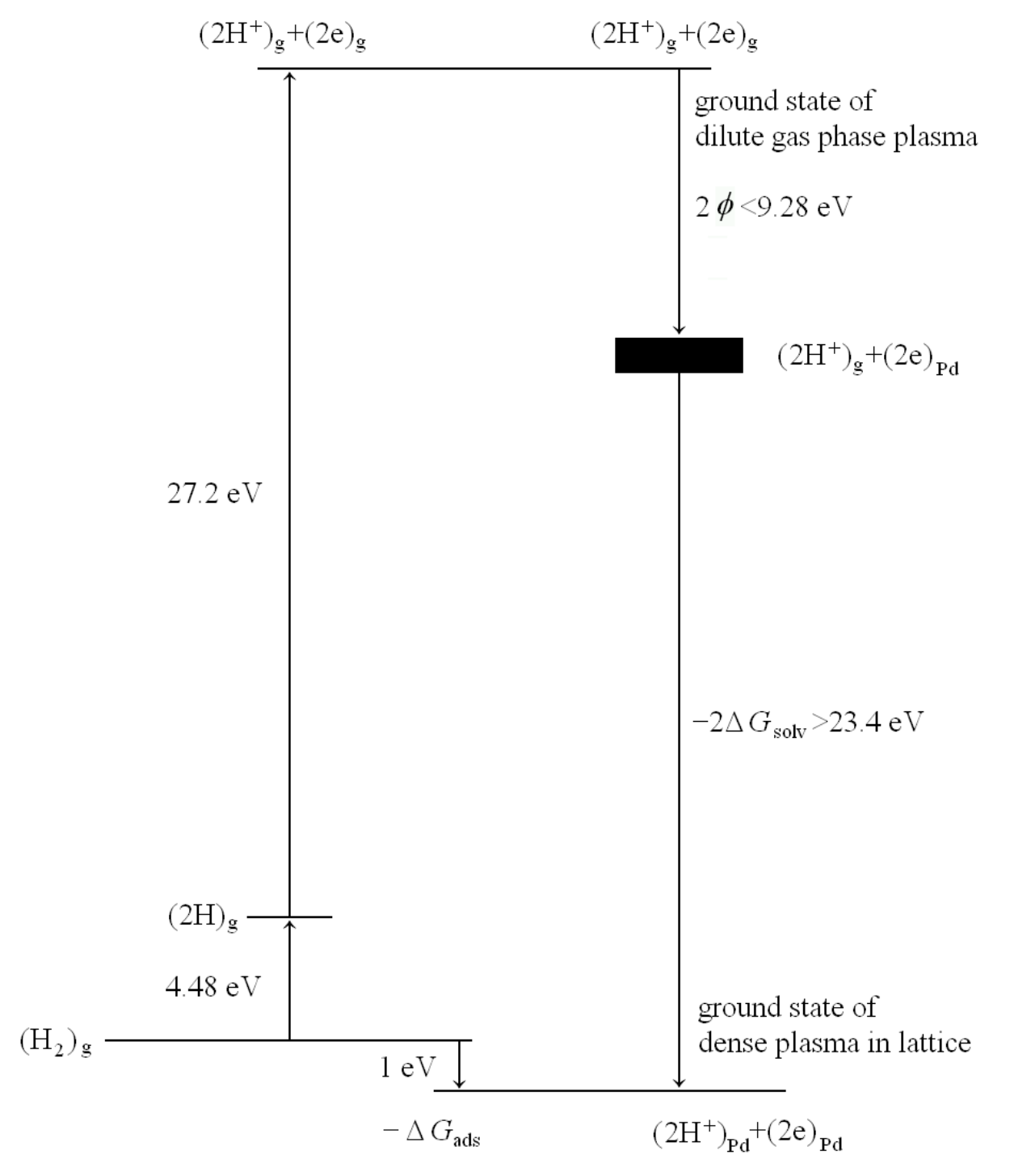

Fig. 1. - Yet another possible Born-Haber cycle for the $\mathbf{H}_{2}$ absorption in metallic Pd.

We draw attention finally to a further consequence of the marked raising of the chemical potential of dissolved hydrogen (deuterium) due to the cathodic loading of the lattice. Our first approach to the problem included the hypothesis that the raising of the chemical potential would lead to the formation of clusters of deuterons dispersed in the palladium lattice. At the very least we would expect that it would lead to the formation of ordered domains having high $\mathrm{D} / \mathrm{Pd}$ ratios.

We summarize the properties and deductions which we have considered in this section in table I which also refers to the possible and impossible models of these systems discussed in sect. 3 .

\section{2. - The phenomenology of «cold fusion».}

Nuclear phenomena induced in metal host lattices have now been observed using three principal methods of approach, fig. 2. Electrochemical loading leads to transient or steady-state effects while transient effects only are observed using loading from the gas phase or by ion implantation followed by changes of temperature. We note here that the transient compression 
induced by such changes of temperature is broadly equivalent to the steady-state compression induced by cathodic polarisation. The important experiments indicated by the dashed arrows in fig. 2 require further exploration and, indeed, there are other possible experiments which have not yet received any attention.

Table I. - Possible and impossible models for the properties of isotopic hydrogen dissolved in palladium host lattices.

\begin{tabular}{lll}
\hline Property & Possible models & Impossible models \\
\hline $\begin{array}{l}\text { Ionised species in } \\
\text { concentration range } \\
60-100 \mathrm{M}\end{array}$ & $\begin{array}{l}\text { Large electrostatic fields to } \\
\text { ionise } H \\
\text { Deep electrostatic holes } \\
\text { collective states of high-density } \\
\text { plasmas }\end{array}$ & Two-particle interactions \\
\hline $\begin{array}{l}\text { Lattice has high proton } \\
\text { affinity }\end{array}$ & $\begin{array}{l}\text { Collective states of macroscopic } \\
\text { quantum system }\end{array}$ & $\begin{array}{l}\text { Behaviour of single particles } \\
\text { deep energy wells of shallow } \\
\text { curvature }\end{array}$ \\
$\begin{array}{l}\text { Protons behave as classical } \\
\text { oscillators }\end{array}$ & & \\
\hline $\begin{array}{l}\text { High diffusion coefficients } \\
\text { Phenomenon }\end{array}$ & Motion of ionised particles & Quasi-particles \\
\hline $\begin{array}{l}\text { Possible models } \\
\begin{array}{l}\text { Inverse isotope effect for } \\
\text { superconductivity }\end{array}\end{array}$ & Properties of collective states & Many \\
\hline $\begin{array}{l}\text { Species reach high chemical } \\
\text { potentials }\end{array}$ & $\begin{array}{l}\text { Clusters of particles on ordered } \\
\text { domains }\end{array}$ & \\
\hline
\end{tabular}

One of the major difficulties of the experiments to date has been the marked variability of the experiments which is certainly in part due to metallurgical factors but mainly caused by variability in the surface chemistry (electrochemistry). While it is possible to make «inspired guesses» as to the causes of the irreproducibility, it is certainly still true that the responsible factors remain to be established.

The generation of medium [10,11,20-22] or of low (see, e.g. [21, 23-25]) levels of ${ }^{3} \mathrm{~T}$ and even lower levels of neutron fluxes [26-50], has now been extensively documented. The fact that the ${ }^{3} \mathrm{~T} / \mathrm{n}$ ratio is very high coupled to the absence of high-energy $\gamma$-radiation and of secondary neutrons [34-36,48] shows clearly that the scenario is not that of the conventional fusion processes of highly energetic deuterons [51],

i) ${ }^{2} \mathrm{D}+{ }^{2} \mathrm{D} \rightarrow{ }^{3} \mathrm{~T}+{ }^{1} \mathrm{H}$,

ii) ${ }^{2} \mathrm{D}+{ }^{2} \mathrm{D} \rightarrow{ }^{3} \mathrm{He}+\mathrm{n}$,

which have roughly equal cross-sections in conventional fusion systems. The observation of 2.45 $\mathrm{MeV}$ neutrons [26,27,30,31] and of energetic protons emitted from thin foil electrodes [52] in 
particle emission experiments shows that the outgoing channels may involve processes of the type i) and ii) but the marked changes in the relative cross-sections show that the lattice profoundly influences the course of the overall processes. This is also shown by the observation of highly energetic particles of mass 3 (probably tritons) from gas-loaded samples [53] and from ion-implanted foils [54] as well as of neutrons in the 4 to $6 \mathrm{MeV}$ range [31]. The «bursts» in the production of tritium and of neutrons $[36,41,42,48,50]$ which have now been well characterised indicate that the observations of these channels may well require the formation of special domains.

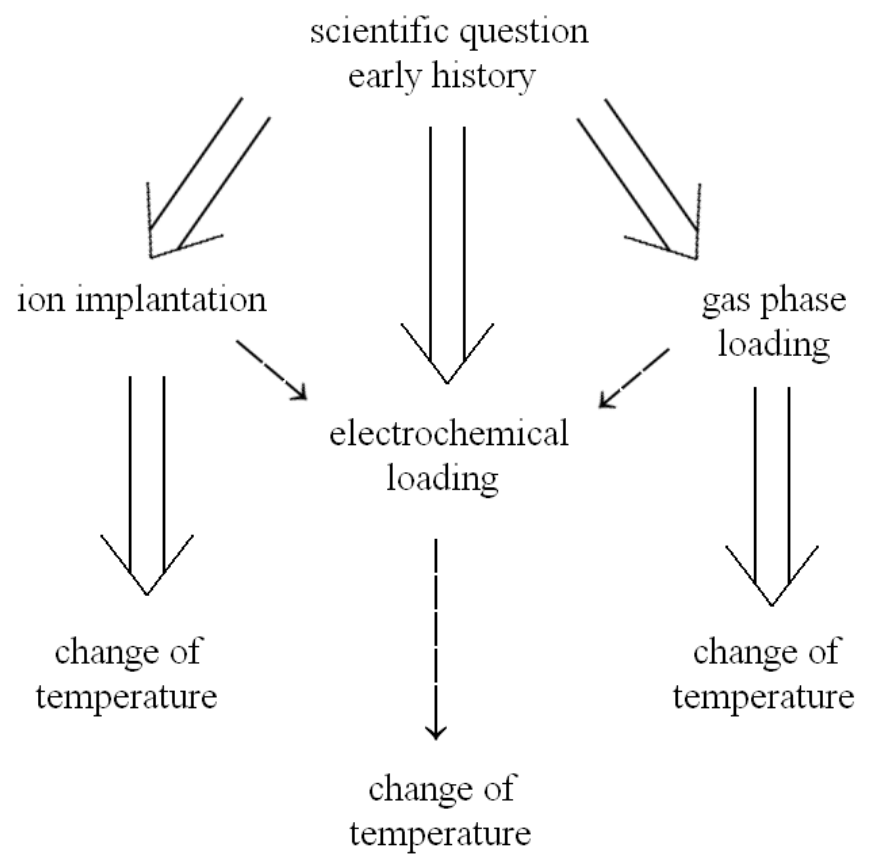

Fig. 2. - The principal methods of approach to nuclear phenomena in metal host lattices.

The generation of characteristic $K_{a}$ and $K_{\beta}$ X-ray radiation of Ti in deuterium-loaded samples subjected to temperature cycles can be attributed to the Coulomb excitation induced by the $\beta$ decay of tritium $[22,55]$ in the lattice. It appears that soft X-rays may also be generated by other Coulomb excitation processes [24] or by the thermalisation of the excited electron plasma (see sect. 4).

The main anomaly of the processes taking place in the lattice is that the sum of all the nuclear products detected does not match the excess enthalpy generated in the cathodes above the one to be attributed to the Joule enthalpy input (e.g. see [7,8,37, 34,35,56-67]). The observation of ${ }^{4} \mathrm{He}$ in the gas phase of cathodes producing excess enthalpy in rough accord with that predicted for the reaction

$$
\text { iii) }{ }^{2} \mathrm{D}+{ }^{2} \mathrm{D} \rightarrow{ }^{4} \mathrm{He}+23.9 \mathrm{MeV} \text {, }
$$

indicates that this is probably the dominant process involved in «cold fusion» [68]. The absence of $\gamma$-radiation indicates a scenario in which there is strong-dipole coupling to the electron plasma rather than to the vacuum fluctuations. 


\section{3. - Possible and impossible models of hydrogen in metals.}

A number of the surprisingly strange properties of hydrogen and its isotopes dissolved in metal host lattices (such as Pd) have been listed in sect. 1 and, according to the scenario outlined at the end of that section, these properties require a reappraisal of the generally accepted picture of condensed-matter physics in deriving satisfactory models for these systems.

The first restriction which must be placed on the modelling of these systems is that an hydrogen atom (or molecule) must find very strong electric fields in order to maintain itself in the ionized state (or to dissociate into two atoms which are then ionized). This means that the electric fields inside the lattice must be able to produce acceleration energies of the order of the Rydberg $(=13.5 \mathrm{eV})$ over distances of the order of the Bohr radius $(=0.57 \AA)$. Clearly this requires a very singular electron distribution! On the other hand we know that ${ }^{4} \mathrm{He}\left(\right.$ or $\left.{ }^{3} \mathrm{He}\right)$ is present in the atomic state in the lattice and this shows that the peculiar charge distribution cannot produce fields of the order $70 \mathrm{eV} / 0.5 \AA$ as, otherwise, the ${ }^{4} \mathrm{He}$ would move through the lattice as an $\alpha$-particle. We will see that this poses important restrictions on the field distribution in the lattice. Furthermore, the dramatic difference in behaviour of hydrogen and its isotopes on the one hand and ${ }^{4} \mathrm{He}$ on the other pre-empts the drawing of any analogy between their metal affinities as has been suggested recently [69]: two separated deuterons are in no sense equivalent to an helium atom.

The necessary conclusion we must draw from these well-established properties is that inside Pd (and, possibly, a number of other metals and alloys) there must be deep potential wells (holes) characterised by electric fields which cannot be lower than about $30 \mathrm{eV} / \AA$ but cannot exceed about $140 \mathrm{eV} / \AA$ In addition, for Pd, the number of such holes must exceed the typical $\beta$-phase concentration ratio $x=\mathrm{H} / \mathrm{Pd}=0.6$. The difficulty of charging the electrodes above $x \approx 1$ makes it plausible that the number of holes just equals the number of $\mathrm{Pd}$ atoms. Here we meet an immediate difficulty: how can we reconcile such deep potential wells, where the hydrogen nuclei would be expected to be strongly bound and performing small oscillations, with the virtually unhindered motion of the species in the lattice (they behave as classical oscillators)? The implication is that even if we can formulate an exotic mechanism which can produce the deep holes required, we then have to invoke a different exotic mechanism to explain why the nuclei do not fall deeply into these holes notwithstanding the fact that this is extremely advantageous from the energetic point of view.

It is our view that the oddity of observations such as these is already sufficient to show that all theories based on the conventional electrostatic picture of condensed matter must be classified as belonging to the category of «impossible theories». The reason is the unresolvable conflict between the existence of deep potential wells and the facile movement of a positively charged heavy particle such as hydrogen and its isotopes.

Before discussing the necessary features of possible theories we recall some further odd properties from sect. 1 . Thus the magnitudes of the diffusion coefficients $D_{\mathrm{D}^{+}}>D_{\mathrm{H}^{+}}>D_{\mathrm{T}^{+}}$ contradict the naive prediction of the isotope effects due to the dominant influence of the mass of the nuclei; the expectations are satisfied for the two fermions $\mathrm{H}^{+}$and $\mathrm{T}^{+}$but do not hold for the bosonic $\mathrm{D}^{+}$. This is another difficulty posed for the generally accepted picture based on the dominance of few-body forces; this difficulty is reinforced by the «inverse» isotope effect on the critical temperatures of highly loaded Pd-hydrides. 
Finally, the very high chemical potentials of hydrogen in metal lattices which can be reached by cathodic loading or by gas phase loading or ion implantation followed by changes of temperature indicate that there are appropriate thermodynamic conditions for the formation of large clusters of hydrogen nuclei or of regions of the lattice containing ordered arrays of hydrogen nuclei at high $\mathrm{H} / \mathrm{Pd}$ ratios. We will see that this point is especially relevant to our discussion.

The comments made above show the necessity of considering collective many-body phenomena.

The deep electrostatic holes (about $100 \mathrm{eV}$ deep and $2 \AA$ wide) that must form in roughly 1:1 proportion to the metal atoms probably originate from coherent plasma oscillations of electrons which belong to bonds which are both localized and, at the same time, not very tightly bound to the nuclei; we note that the d-electrons of Pd and of other metals that have high affinities for hydrogen (and where hydrogen is present as protons) appear to share this characteristic. In fact, the localisation is required to create a lattice of holes, while the relatively weak binding is necessary to allow plasma oscillations of large amplitudes $(\sim 0.5 \AA)$. We note here that this is just the situation that was proposed for the Pd-lattice namely, that $100 \mathrm{eV}$ deep holes could be generated by oscillations of the $d$-electrons arranged in a lattice between the tetrahedral positions [15].

We consider now the difficult problem of hydrogen delocalisation inside the lattice of deep electrostatic holes. As delocalisation depends on the occupancy by the protons of highly excited states of the well, this configuration must be energetically advantageous. It then becomes clear that collective phenomena must come into play as, otherwise, the hydrogen nuclei $\left(\mathrm{H}^{+}, \mathrm{D}^{+}\right.$or $\left.\mathrm{T}^{+}\right)$ could not avoid going into the ground state. The many-body interactions of the hydrogen nuclei must therefore be able to supply the energy required to raise the nuclei to highly excited states of oscillation. It is again evident that this cannot be achieved through short-range forces, thus providing another clear illustration of the inadequacy of conventional theories. On the other hand, the superradiant plasma of hydrogen nuclei considered elsewhere [70] leads immediately to such highly excited states of the oscillating nuclei by virtue of the superradiant behaviour of the ideal plasma: the highly excited states of the oscillating nuclei compensate their high kinetic energy by the interaction energy with the coherent superradiant electromagnetic field. If this is kept in mind, then one can readily understand the odd properties of $\mathrm{H}$ in $\mathrm{Pd}$ : thus the high diffusion coefficients reflect the «quasi-free» character of the hydrogen «band» in the lattice of deep holes; the inverse isotope effects of the diffusion coefficients and of the critical temperatures for transition to the superconducting states are due to the bosonic character of $\mathrm{D}^{+}$as opposed to the fermionic character of $\mathrm{H}^{+}$and $\mathrm{T}^{+}$, the Pauli principle restricting the configuration space of $\mathrm{H}^{+}$and $\mathrm{T}^{+}$but not of $\mathrm{D}^{+}$. Finally, the high chemical potentials are a likely consequence of the formation of clusters in the size range of a few microns, the size of the coherence domains of hydrogen plasmas [15]. A further aspect of the superradiant behaviour of these systems is referred to in sect. 5 .

\section{4. - Conditions for possible theories of «cold fusion».}

The main aspects of the phenomenology of «cold fusion» that have been determined up to the present time have been outlined in sect. 2 . As has been stressed previously, the two difficult problems that must be dealt with by any theory of these phenomena are: 
i) how can the Coulomb barrier be penetrated at such large rates?

ii) how can the processes avoid the restrictions posed by «asymptotic freedom»?

Indeed, in the absence of intense thermal agitation, the two deuterons must come within $10 \mathrm{fm}$ of each other as, otherwise, the short-range forces cannot act and, to reach such positions, the deuterons must have «tunnelled» over long distances (starting from separations of $\approx 1 \AA$ ) through a singular barrier such as the Coulomb barrier. All simple evaluations of such «tunnelling» probabilities lead to estimates that are 50-60 orders of magnitude too small. Even if this severe difficulty could be resolved one immediately is confronted with an even more severe difficulty, namely, that fusion clearly does not proceed in the same way as in the high-energy systems where the two fusion channels

i) ${ }^{2} \mathrm{D}+{ }^{2} \mathrm{D} \rightarrow{ }^{3} \mathrm{~T}(1.01 \mathrm{MeV})+{ }^{1} \mathrm{H}(3.02 \mathrm{MeV})$,

ii) ${ }^{2} \mathrm{D}+{ }^{2} \mathrm{D} \rightarrow{ }^{3} \mathrm{He}(0.82 \mathrm{MeV})+\mathrm{n}(2.45 \mathrm{MeV})$,

have roughly equal cross-sections. It is the observed large ratio between the rate of excess enthalpy production and that predicted on the basis of the observed rates of reaction ii) and, also, the observed large ratio of reaction ii) compared to i) that shows that DD fusion in the host lattice must take place in a completely different way from that, say, in a gas at low pressures. Thus the nuclei cannot be considered to be «asymptotically free» from lattice effects, «asymptotic freedom» being the picture expected on the basis of conventional nuclear physics and generally accepted condensed matter.

Apart from the resolution of these two fundamental problems, a useful theory should also give some explanation of other less serious but, nevertheless, practically important problems such as the dependence of the fusion rate on the loading ratio $x$, the variability of the phenomena, the observation of «bursts» in ${ }^{3} \mathrm{~T}$ and $\mathrm{n}$, which appear to be primarily connected with the way deuterons are distributed in the lattice and of the generation of high-energy $(\sim 5 \mathrm{MeV})$ neutrons and particles of mass 3 . We will discuss these problems in sequence:

\section{i) Penetration of the Coulomb barrier.}

A simple calculation shows that a screening of the Coulomb repulsion potential by a negative energy of about $100 \mathrm{eV}$ is sufficient to enhance naive estimates of the fusion rates by 50 orders of magnitude thereby giving rates compatible with the rates of neutron production [14]. The observation that this energy is of the same magnitude as the depth of the holes which we have argued to be necessary to describe the phenomenology of hydrogen in $\mathrm{Pd}$ is reassuring. We note here that possible theories of cold fusion must always include at the very least a semiquantitative explicit explanation and estimation of the effects of screening potentials in reducing the Coulomb barrier, since it is this barrier which must be penetrated no matter what finer details may be imposed on the model. Thus, in our view, it is not permissible to ignore the barrier [71], to believe that it somehow becomes irrelevant when the deuterons are arranged in a lattice [72] or when some resonance condition is obeyed [73] or when there is some combination of these situations. We note here that we have specified that one should demand only a semi-quantitative estimate at this stage of the development of the subject: more exact estimates will no doubt become possible when the effects of the many parameters affecting the various signatures are understood so that the models can be specified more closely.

A further possible way of avoiding the Coulomb repulsion is the proposal that fusion takes place between two particles one of which is either neutral (a neutron) [74] or seen as neutral by 
the other particle down to very short distances $(\sim 300 \mathrm{fm})$ [75]. We regard these proposals as being impossible unless one is able to show that «on shell» neutrons can be produced from the deuterons in the lattice, or that electrons can stick to deuterons at distances as small as a few hundred fm. The latter proposal [75], in particular, appears to be in conflict with the known phenomenology of deuterium atoms which have never been observed to form any narrow resonances at a few eVs above the continuum threshold.

\section{ii) The violation of «asymptotic freedom».}

It should be self-evident that in a lattice composed of short-range forces there is absolutely no room for invoking such striking phenomena, for at the distances and times characteristic of nuclear phenomena $\left(10^{-12} \mathrm{~cm}\right.$ and $\left.10^{-21} \mathrm{~s}\right)$ the lattice times and distances $\left(10^{-8} \mathrm{~cm}\right.$ and $\left.10^{-15} \mathrm{~s}\right)$ become totally irrelevant. It is the collective phenomena which change the times and spaces involved and which lead to the influence of lattice effects on nuclear processes. We note here that this is equally true of Mössbauer spectroscopy [76] as of «cold fusion»: possible theories of «cold fusion» therefore must take due account of such collective effects.

We recall here that the theoretical analysis based on the superradiant behaviour of the deuteron and $d$-electron plasmas $[14,15]$ explicitly violates asymptotic freedom and provides estimates of

the rates of ${ }^{4} \mathrm{He}$ (in excess heat), ${ }^{3} \mathrm{~T}$ and n-production which are in reasonable agreement with the known phenomenology. In particular, the production of ${ }^{4} \mathrm{He}$ unaccompanied by high-energy $\gamma$ rays [68] demands a very fast $\left(\sim 10^{-21} \mathrm{~s}\right)$ transfer of energy to the electrons of the lattice that will then relax in various ways: heat and electromagnetic radiation in a broad range of frequencies including soft X-rays. The impossibility of accounting for this by conventional theories can be well appreciated by estimating the velocity with which energy must «travel» through the lattice. Setting the lattice constant at $\alpha \approx 3 \AA$ one has $v=a / \Delta t \approx 10^{3} \mathrm{c}$. This absurdity reminds us of the Einstein-Podolski-Rosen paradox, where superluminal propagation seems to be implied by the erroneous interpretation of a quantum phenomenon. Indeed, if in the present case the systems between which energy transfer takes place, the deuterons and the $d$-electrons, are both in macroscopic quantum states, then the estimate above of the velocity of energy transfer completely loses any meaning.

iii) Variability, bursts, etc.

The observed erratic behaviour of «cold fusion» phenomena has been related to the existence of a new very heavy charged particle that might be present in showers that pour irregularly into the earth from outer space [77]. Although such theories are not impossible, we prefer to explore fully the implications of conventional physics (even though this does require the full acceptance of QED in a lattice!) before resorting to more exotic explanations.

In this vein, possible theories for such sporadicity include threshold effects in $x$ at $x \approx 1$ at which point there is a full occupancy of the lattice of deep hole deuterons [15]. Above this loading the deuterons may access rather rare fusion channels instead of falling into the remaining holes. The regions of space where this takes place would be expected to be unevenly distributed and to vary in time. Lattice inhomogeneities may also be responsible for tritium and neutron bursts, the latter in conjunction with the formation of fractures since intense thermal and mechanical stresses can be established $[15,78]$. 
iv) High-energy neutrons, protons and particles of mass 3.

The only possible explanation of the observation of nuclear particles with atypically high energies appears to be that of many-body fusion, such as DDD. The particular mechanisms by which this may happen still await clarification. However, here again we can say that possible explanations of such phenomena must involve collective processes both in the deuteron and $d$-electron plasmas as, otherwise, the Coulomb barriers would be quite prohibitive.

We have summarized the above arguments in table II.

Table II. - Possible and impossible models for the explanation of nuclear phenomena in deuterium-loaded lattices.

\begin{tabular}{|c|c|c|}
\hline Phenomenon & Possible models & Impossible models \\
\hline $\begin{array}{l}\text { Dependence on electrochemical } \\
\text { loading or ion implanation or gas } \\
\text { loading coupled to changes of } \\
\text { temperature }\end{array}$ & $\begin{array}{l}\text { Stationary or transient } \\
\text { compression of } \mathrm{D}^{+} \text {in the } \\
\text { lattice; formation of ordered } \\
\text { arrays }\end{array}$ & Many \\
\hline Variability of the phenomena & $\begin{array}{l}\text { Dependence on surface } \\
\text { chemistry/electrochemistry as } \\
\text { well as metallurgy; state of } \\
\text { system is not a state function } \\
\text { of the system variables }\end{array}$ & $\begin{array}{l}\text { Strange particles; quasi- } \\
\text { particles }\end{array}$ \\
\hline $\begin{array}{l}\text { Low levels of }{ }^{3} \text { T production; low } \\
\text { levels of } n \text { production; } \mathrm{T} / \mathrm{n} \gg 1 \text {; } \\
\text { absence of high-energy } \gamma \text {-rays; } \\
\text { absence of secondary neutrons }\end{array}$ & $\begin{array}{l}\text { e.m. coupling to electrons in } \\
\text { outgoing channel }\end{array}$ & $\begin{array}{l}\text { The conventional two-body } \\
\text { fusion routes }\end{array}$ \\
\hline Bursts in ${ }^{3} \mathrm{~T}$ and $\mathrm{n}$ production & Creation of special domains & \\
\hline $\begin{array}{l}\text { High-energy tritons, neutrons and } \\
\text { protons }\end{array}$ & $\begin{array}{l}\text { Three-body reactions (or } \\
\text { many body reactions) in } \\
\text { clusters }\end{array}$ & $\begin{array}{l}\text { Conventional theories of } \\
\text { fusion }\end{array}$ \\
\hline Soft X-rays & $\begin{array}{l}\text { Coulomb excitation due to } \\
\beta \text {-decay of }{ }^{3} \mathrm{~T} \text {; excitation of } \\
\text { electrons of host lattice }\end{array}$ & \\
\hline${ }^{4} \mathrm{He}$ in the absence of $\gamma$-rays & $\begin{array}{l}\text { Excitation of electrons of host } \\
\text { lattice }\end{array}$ & \\
\hline $\begin{array}{l}\text { Excess enthalpy production far in } \\
\text { excess of that corresponding to } T+n \\
\text { yield }\end{array}$ & $\begin{array}{l}\text { High cross-section of D-D } \\
\text { reaction requires many-body } \\
\text { explanation of screening of } \\
\text { Coulomb repulsion and } \\
\text { coherent scattering }\end{array}$ & \\
\hline
\end{tabular}




\section{5. - Conclusions.}

We have shown that satisfactory explanations of the conventional behaviour of hydrogen and its isotopes dissolved in metal host lattices as well as of the phenomenology of «cold» fusion must be based on models which take full account of the collective behaviour of the proton (deuteron) and electron plasmas. As far as «cold fusion» is concerned this leads to ready explanations both of the reduction of the Coulomb barriers as well as of the violation of the principle of «asymptotic freedom».

A key feature of these models is that the superradiant proton (deuteron) and electron plasmas are described by macroscopic wave functions whose coupling can account both for the creation of the required initial state to allow deuteron tunnelling and the dissipation of the energy in the outgoing channel. Such an argument should not come as a surprise to physical chemists at least since there is some analogy between the deuteron-electron plasma coupling and the dipolefluctuation-induced activation of outer-sphere redox reactions. Indeed it is our view that the latter processes would be best described by the relevant macroscopic wave functions of that superradiant system. We note also that reactions at metal surfaces could well be described by the macroscopic wave functions which allow for the coupling of the reacting species to the collective modes of the electron plasmas. In the systems we have considered here, a model of this kind would give an immediate explanation for the formation of the «unbound transition state» [3] in the electrochemical discharge of hydrogen and deuterium at Pd-cathodes which we have referred in sect. 1 .

\section{REFERENCES}

1. F. A. Lewis: The Palladium Hydrogen System (Academic Press, London, 1967).

2. W. M. Muller, J. P. Blackledge and G. G. Libowitz: Metal Hydrides (Academic Press, New York, N.Y. 1968).

3. B. Dandapani and M. Fleischmann: J. Electroanal. Chem., 39, 323 (1972).

4. J. Voelkl and G. Alefeld (Editors): Hydrogen and Metals, Vol. 2, in Topics in Applied Physics, Vol. 29 (Springer-Verlag, Berlin, 1978), Chapt. 12.

5. G. Bambakadis (Editor): Metal Hydrides (Plenum Press, New York, N.Y., 1981).

6. L. Schlapbach (Editor): Hydrogen in Intermetallic Compounds, Vol. 1, in Topics in Applied Physics, Vol. 63 (Springer-Verlag, Berlin, Heidelberg, 1988).

7. M. Fleischmann, S. Pons and M. Hawkins: J. Electroanal. Chem., 261, 301 (1989); for some corrections see J. Electroanal. Chem., 263, 187 (1989).

8. M. Fleischmann, S. Pons, M. W. Anderson, L. J. Li and M. Hawkins: J. Electroanal. Chem., 287, 293 (1990).

9. S. Pons and M. Fleischmann: Fusion Technol., 17, 669 (1990).

10. J.O’M. Bockeis, G. H. Lin and N. J. C. Packham: Fusion Technol., 18, 11 (1990).

11. J. O’M. Bockris and D. Hodko: Chem Indust., 21, 688 (1990).

12. M. Srinivasan: Current Sci, April 1991.

13. E. Storms: Fusion. Technol., 20, 433 (1991).

14. T. Bressani, E. Del Giudice and G. Preparata: Nuovo Cimento A, 101, 845 (1989).

15. G. Preparata: Proceedings of the First Annual Conference on Cold Fusion, Salt Lake City, Utah, March 1990, p. 91. 
16. A. Coehn: Z. Electrochem., 35, 676 (1929); A Coehn and W. Specht: Z. Phys., 62, 1 (1930); A. Coehn and H. Jurgens: Z. Phys., 71, 179 (1931); A Coehn and K. Sperling: Z. Phys., 83, 291 (1933).

17. A. H. Verbraggen, R. Griessen and J. H. Rector: Phys. Rev. Lett., 52, 1625 (1986).

18. T. Soskiewicz: Phys. Status Solidi A, 11, k123 (1972); B. Stritzker and W. Buckl: Z. Phys., 257, 1 (1972).

19. T. B. Flanagan and J. F. Lynch: J. Phys. Chem., 79, 444 (1975).

20. N. Packham, K. L. Wolf, J. L. Wass, R. L. Kainthla and J. O’M. Bockris: J. Electroanal. Chem., 270, 451 (1989).

21. P. K. Iyengar and M. Srinivasan: Proceedings of the First Annual Conference on Cold Fusion, Salt Lake City, Utah, March 1990, p. 62.

22. T. C. Kanshik, A. Shyam, M. Srinivasan, R. K. Rout, L. V. Kulkarni, M. S. Krishnan, S. K. Malhotra and V. B. Naguenkar: Indian J. Technol, 28, 667 (1990).

23. E. Storms and C. Talcott: Fusion Technol, 17, 680 (1990).

24. S. Szpak, P. A. Mosier-Boss and J. J. Smith: J. Electroanal. Chem., 302, 255 (1991).

25. T. N. Claytor, P. A. Seeger, R. K. Rohwer, D. G. Tuggle and W. R. Doty: NSF/EPRI Conference Washington D.C., October 1989; see also Proceedings of the Anomalous Nuclear Effects in Deuterium Systems, Provo, Utah, October 1990.

26. J. E. Jones, E. P. Palmer, J. B. Czirr, D. L. Decker, G. L. Jensen, J. M. Thorne, S. F. Taylor and J. Rafelski: Nature, 338, 737 (1989).

27. K. L. Wolf, N. J. C. Packman, D. Lawson, J. Shoemaker, F. Cheng and J. C. Wass: J. Fusion Energy, 9, 105 (1990).

28. A. Bertin, M. Bruschi, M. Capponi, S. De Castro, V. Marconi, C. Moroni, M. Piccinini, N. Semprini-Cesari, A. Trombini, A. Vitale, A. Zoccoli, S. E. Jones, J. B. Czirr, G. L. Jensen and E. P. Palmer: Nuovo Cimento A, 101, 997 (1989).

29. S. E. Jones, E. P. Palmer, J. B. Czirr, D. L. Decker, G. L. Jensen, J. M. Thorne, S. F. Taylor and J. Rafelski: J. Fusion Energy, 9, 199 (1990).

30. A. Takahashi, T. Iida, F. Maekawa, H. Sugimoto and S. Yoshida: Fusion Technol, 19, 380 (1991).

31. A. Takahashi, T. Takeuchi and T. Iida: J. Nucl Sci. Technol, 27, 663 (1990).

32. F. Celani, A. Spallone, S. Pace, B. Polichetti, A. Saggese, L. Liberatori, V. Di Stefano and P. Marini: Fusion Technol, 17, 718 (1990).

33. A. de Ninno, A. Frattolillo, G. Lollobattista, A. Martinis, M. Martone, L. Mori, S. Podda and F. Scaramuzzi: Nuovo Cimento A, 101, 841 (1989).

34. D. Gozzi, P. L. Cignini, L. Petrucci, M. Tomellini and G. De Maria: Nuovo Cimento A, 103, 143 (1990).

35. D. Gozzi, P. L. Cignini, L. Petrucci, M. Tomellini, G. De Maria, S. Frullani, F. Garibaldi, F. Ghio, M. Jodice and E. Tabet: J. Fusion Energy, 9, 241 (1990).

36. P. K. Iyengar et al: Fusion Technol, 18, 32 (1990).

37. D. Lewis and K. Skoeld: J. Electroanal. Chem., 294, 275 (1990).

38. T. Lin and C. Lin: J. Fusion Energy, 9, 487 (1990).

39. A. G. Lipson, D. M. Sakov, V. A. Klyuev, B. V. Deryagin and Yu. P. Toporov: JEPT Lett, 49, 675 (1989).

40. B. V. Deryagin, A. G. Lipson, V. A. Klyuev, D. M. Sakov and Yu. P. Toporov: Nature, 341, 492 (1989). 
41. H. O. Menlove, M. M. Fowler, E. Garcia, A. Mayer, M. C. Miller, R. R. Ryan and S. E. Jones: J. Fusion Energy, 9, 215 (1990).

42. H. O. Menlove, M. M. Fowler, E. Garcia, M. C. Miller, M. A. Paciotti, R. R. Ryan and S. E. Jones: J. Fusion Energy, 9, 495 (1990).

43. E. Yamaguchi and T. Nishioka: Jpn. J. Appl Phys., 29, L666 (1990).

44. T. Izumida, Y. Ozawa, K. Ozawa, S. Izumi, S. Uchida, T. Miyamoto, H. Yamashita and H. Miyadera: Fusion Technol, 18, 641 (1990).

45. P. G. Sona, F. Parmigiani, F. Barberis, A. Battaglia, R. Berti, G. Buzzanca, A. Cappelli, D. Capra and M. Ferrari: Fusion Technol, 17, 713 (1990).

46. P. Perfetti, F. Cilloco, R. Felici and M. Capozi: Nuovo Cimento D, 11, 921 (1989).

47. Y. Arata and Y. Zang: Fusion Technol, 18, 95 (1990).

48. C. Sanchez, J. Sevilla, B. Escarpizo, F. J. Fernandez and J. Canizares: Solid StateCommun., 71, 1039 (1989).

49. J. Sevilla, F. Fernandez, B. Escarpizo and C. Sanchez: Fusion Technol., 19, 188 (1991).

50. T. Sato, M. Okamoto, P. Okamoto, P. Kim, Y. Fujiu and O. Aizawa: Fusion Technol., 19, 357 (1991).

51. M. L. Oliphant, P. Hartech and Lord E. Rutherford: Nature, 133, 413 (1934).

52. R. Taniguchi, T. Yamamoto and S. Irie: Jp. J. Appl. Phys., 28, L2021 (1989).

53. F. E. Cecil, D. Ferg, T. E. Furtak, C. Mader, J. A. Mc Neil and D. L. Williamson: J. Fusion Energy, 9, 195 (1990).

54. G. P. Chambers, G. K. Hubler and K. S. Grabowski: Proceedings of the Anomalous Nuclear Effects in Deuterium/Solid Systems, Provo, Utah, October 1990.

55. P. K. Iyengar et al: Fusion Technol., 18, 32 (1990).

56. A. J. Appleby, Y. J. Kim, O. J. Murphy and S. Srinivasan: Proceedings of the First Annual Conference on Cold Fusion, Salt Lake City, Utah, March 1990, p. 32.

57. A. Arata and Y. Zhang: Fusion Technol., 18, 95 (1990).

58. R. T. Bush and R. D. Eagleton: J. Fusion Energy, 9, 397 (1990).

59. D. P. Hutchinson, U. A. Bennett, R. K. Richards, J. Bullock IV and G. L. Powell: Proceedings of the First Annual Conference on Cold Fusion, Salt Lake City, Utah, March 1990.

60. R. C. Kainthla, O. A. Velev, L. Kaba, U. H. Lin, N. Packham, J. O’M. Bockris, M. Szkarczylc and J. Wass: Electrochem. Acta, 34, 1315 (1989).

61. M. C. H. McKubre, R. C. Rocha-Filho, S. Smedley, F. Tanzella, J. Chao, B. Chexal, T. Passell and J. Santucci: Proceedings of the First Annual Conference on Cold Fusion, Salt Lake City, Utah, March 1990, p. 20.

62. M. H. Miles, K. H. Park and D. E. Stilwell: J. Electroanal. Chem., 296, 241 (1990).

63. V. C Nonniski and C. I. Nonniski: Fusion Technol., 19, 364 (1991).

64. R. A. Oriani, J. C. Nelson, S. Lee and J. H. Broadhurst: Fusion Technol., 18, 652 (1990).

65. K. S. V. Santhanam, J. Rangarajan, O’N. Braganza, S. K. Haram, N. M. Limaye and C. K. Mandal: Indian J. Technol., 27, 175 (1989).

66. T. M. Gur, M. Schreiber, G. Lucier, J. A. Ferrante, J. Chao and R. A. Huggins: Proceedings of the First Annual Conference on Cold Fusion, Salt Lake City, Utah, March 1990, p. 82.

67. C. D. Scott, J. E. Mecochez, T. C. Scott, G. E. Michaels, E. Neuruan and M. Petek: Fusion Technol., 18, 103 (1990).

68. B. F. Bush, J. J. Lagowski, M. A. Miles and G. S. Ostrom: J. Electroanal. Chem., 304, 271 (1991). 
69. A. J. Leggett and G. Baym: Nature, 340, 45 (1989).

70. G. Preparata: Quantum field theory of superradiance, in Problems of Fundamental Modern Physics, edited by R. Cherubini, P. Dal Piaz and B. Minetti (World Scientific, Singapore, 1990).

71. J. Schwinger: Proceedings of the First Annual Conference on Cold Fusion, Salt Lake City, Utah, March 1990, p. 130.

72. S. R. Chubb and T. A. Chubb: Proceedings of the First Annual Conference on Cold Fusion, Salt Lake City, Utah, March 1990, p. 119.

73. R. T. Bush: Fusion Technol., 19, 313 (1991).

74. P. Hagelstein: Proceedings of the First Annual Conference on Cold Fusion, Salt Lake City, Utah, March 1990, p. 99.

75. F. J. Meyer and J. R. Reitz: Fusion Technol., 19, 552 (1991).

76. T. Bressani, E. Del Giudice and G. Preparata: Nuovo Cimento D, 14, 345 (1992).

77. J. Rafelski et al: Fusion Technol., 18, 136 (1990).

78. G. Preparata: Nuovo Cimento A, 104, 1259 (1991). 